\title{
Genetic approach supports cardiovascular safety of GLP1R agonists
}

A proof-of-concept study has demonstrated that identifying genetic variants that mimic the effects of a drug on its target, and then testing the association of these variants with disease outcomes in large patient cohorts can provide evidence of efficacy and possible adverse effects of novel therapies. "Generating this knowledge early in the process of drug development is critical to focus investment on the most appropriate targets," says Nick Wareham, a senior investigator of the study.

Wareham and colleagues examined the association of 121 variants in six genes encoding drug targets for type 2 diabetes or obesity (CNR2, DPP4, GLP1R, SLC5A1, HTR2C, and MCHR1) with variation in several metabolic traits, such as BMI and fasting glucose. In an initial analysis in 11,806 individuals, seven variants potentially associated with metabolic traits were identified through targeted exome sequencing. These seven variants were then investigated by targeted genotyping in 39,979 individuals.

In a combined analysis, the investigators identified a low-frequency missense variant in the gene encoding the glucagon-like peptide 1 receptor (GLP1R) that was associated with lower fasting glucose and lower risk of type 2 diabetes, similar to the effects of GLP1R agonists, a widely used therapy for type 2 diabetes and obesity. In an international collaboration that included 61,846 patients with coronary heart disease (CHD) and 163,728 controls, the investigators found that this GLP1R variant was also associated with a lower risk of CHD, which supports the cardiovascular safety of GLP1R agonists and suggests that these drugs might even be cardioprotective.

Randomized clinical trials to assess the cardiovascular safety of GLP1R agonists are underway. "Our genetic approach," says Wareham, "does not preclude the need for randomized controlled trials ... but could provide useful evidence at an early stage ... about designing and investing in such trials."

Irene Fernández-Ruiz

ORIGINAL ARTICLE Scott, R. A et al. A genomic approach to therapeutic target validation identifies a glucose-lowering GLP1R variant protective for coronary heart disease. Sci. Transl. Med. 8, 341ra76 (2016)

FURTHER READING Jukema, J. W. \& Trompet, S.

Genetics: Genetic risk scores-new promises for drug evaluation. Nat. Rev. Cardiol. 12, 321-322 (2015)|Pereira, N. L., Sargent, D. J., Farkouh, M. E. \& Rihal, C. S. Genotype-based clinical trials in cardiovascular disease. Nat. Rev. Cardiol. 12, 475-487 (2015) 